\title{
Estudio epidemiológico de la prevalencia y evolución de hemoparásitos en burros del norte de Nicaragua
}

\section{Epidemiological study of the prevalence and evolution of hemoparasites of donkeys from the north of Nicaragua}

\author{
Enrique Rimbaud Giambruno', Maryuri Inés Mayorga-Escobar², Daviana González ${ }^{3}$, Emilio José Sequeira Valle ${ }^{3}$, \\ Hazel Torres ${ }^{4}$, Carolina Montoya ${ }^{4}$, Sergio Ramírez ${ }^{4}$ \\ DMV, Presidente Fundación A.Mar.Te., Decano de la Facultad de Ciencias Agrarias de la UCC, ORCID: https://orcid.org/0000-0003-3142-8862 \\ ${ }^{2}$ DMV, Directora del Centro de Diagnóstico Veterinario de la Facultad de Ciencias Agrarias de la UCC, ORCID: https://orcid.org/0000-0002- \\ $5350-1496$ \\ ${ }^{3}$ DMV, Veterinario de la Fundación A.Mar.Te. ORCID: https://orcid.org/0000-0003-1594-9561 / ORCID: https://orcid.org/0000-0002-8132- \\ 7747
}

${ }^{4}$ BVSc, Estudiante de 5to año de la carrera de Medicina Veterinaria de la Facultad de Ciencias Agrarias de la UCC

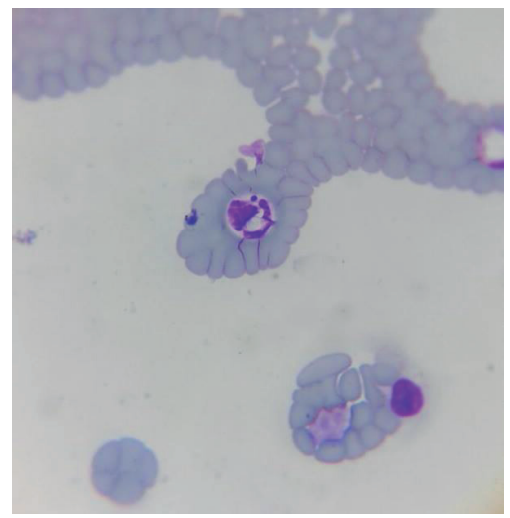

\section{RESUMEN}

El objetivo de este trabajo es comparar los resultados relevados y obtenidos en el 2016, con los obtenidos en el 2017, evaluando la evolución de las enfermedades por hemoparásitos en los burros de la región. Los hemoparásitos son el grupo de enfermedades más importante en burros de la región norte de Nicaragua (Departamentos de Madriz y Nueva Segovia), con una prevalencia cercana al 50\% de los burros existentes. Sin embargo, las causas de muerte se deben mayormente a la falta de control de garrapatas en burros por parte de sus dueños, sumado a la falta de control y tratamiento de la hemoparasitosis. Con el fallecimiento de los burros afectados por Babesia sp., la Anaplasmosis pasa a ser la enfermedad más relevante dentro de los hematozoarios, con más de $30 \%$ de prevalencia. La presencia de Plasmodium sp., no descrito hasta el momento en la literatura internacional es relevante, no solo porque afecta al $16.88 \%$ de los burros de las diferentes comarcas de la cuenca del Río Tapacale en Somoto, sino por los riesgos inherentes a la salud pública. Se precisa en forma urgente capacitar a los dueños de los burros sobre control y tratamiento de garrapatas y hemoparásitos, así como conseguir los fondos para costear los tratamientos.

Palabras clave: Madriz, Nueva Segovia, Babesia sp., Anaplasma sp, Ehrlichia sp, Plasmodium sp, epidemiología.

\section{ABSTRACT}

The objective of this work is to compare the results obtained and obtained in 2016, with those obtained in 2017, evaluating the evolution of hemoparasitic diseases in donkeys in the region. Hemoparasites are the most important group of diseases in donkeys in the northern region of Nicaragua (Departments of Madriz and Nueva Segovia), with a prevalence of around $50 \%$ of the existing donkeys. However, the causes of death are mainly due to the lack of control of ticks on donkeys by their owners, added to the lack of control and treatment of hemoparasitosis. With the death of the donkeys affected by Babesia sp., Anaplasmosis becomes the most relevant disease within the hematozoa, with more than $30 \%$ prevalence. The presence of Plasmodium sp., Not described so far in the international literature is relevant, not only because it affects $16.88 \%$ of the donkeys of the different regions of the Tapacale River basin in Somoto, but because of the risks inherent to health public. It is urgent to train the owners of the donkeys on control and treatment of ticks and hemoparasites, as well as to obtain the funds to pay for the treatments.

Keywords: Madriz, Nueva Segovia, Babesia sp, Anaplasma sp, Ehrlichia sp, Plasmodium sp, epidemiology.
Recibido: 16 de febrero del 2018 Aceptado: 31 de mayo del 2018
Los artículos de la revista La Calera de la Universidad Nacional Agraria, Nicaragua, se comparten bajo términos de la licencia Creative Commons: Reconocimiento, No Comercial, Compartir Igual. Las autorizaciones adicionales a las aquí delimitadas se pueden obtener en el correo freddy.aleman $@$ ci.una.edu.ni

C) Copyright 2018. Universidad Nacional Agraria 


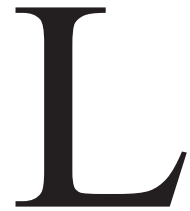

a población y existencia de burros en el norte nicaragüense, sobre todo en los departamentos de Madriz y Nueva Segovia, tienen especial importancia y relevancia, tanto social como económica, dado que son la base fundamental del sustento de las familias campesinas que viven en condiciones de pobreza extrema, tanto por sus tareas de acarreo, carga, arado, transporte y turismo (Rimbaud, et al., 2016; Rimbaud, 2015; Díaz y Talavera, 2017).

En el año 2015, en un brote de mortandad en burros, en Sonys, Somoto, Madriz, encontramos que el causante de la misma había sido el hemoparásito Babesia $\mathrm{sp}$, registrándose que los dueños de burro, no asociaban al mismo con la infestación de garrapatas, y tampoco conocían o sabían como enfrentar el problema con tratamientos adecuados ${ }^{1}$.

Esto llevó a que se realizaran trabajos de investigación para estudiar la prevalencia y epidemiología de estas enfermedades durante los años 2016 y 2017, evaluando la dimensión y envergadura del problema en la región (Rimbaud et al., 2017; Rimbaud, 2015).

El objetivo de este trabajo es comparar los resultados relevados y obtenidos en el 2016, con los obtenidos en el 2017, evaluando la evolución de las enfermedades por hemoparásitos en los burros de la región.

\section{MATERIAL Y MÉTODOS}

En ambos relevamientos se recorrieron las mismas comunidades de ambos departamentos, 16 comunidades en Madriz y 14 comunidades en Nueva Segovia, con la colaboración en Madriz de la Alcaldía de Somoto y la organización no gubernamental Los Burritos de Somoto, y en Nueva Segovia, con la colaboración de las organizaciones no gubernamentales Comisión Ciudad Segovia y Coordinadora de Pueblos Indígenas Chorotegas.

En cada comunidad se relevó el cien por ciento de los animales, realizando encuestas semi estructuradas a los dueños de los burros, extrayendo muestras de todos los animales de la vena de la oreja, la que fue procesada en el laboratorio para el diagnóstico de hemoparásitos mediante la tinción de Diff-Quick (Rodak, 2004).

Las diferencias de cifras encontradas fueron tan contundentes, que no se realizó confrontación estadística, sino que se analizaron por probabilística simple.

Se realizó una encuesta telefónica entre aquellos casos que habían mostrado resultados positivos en el año 2016 y no se presentaron en el año 2017, para determinar las causas.

\section{RESULTADOS}

En el cuadro 1 y 2 se presentan los resultados sobre presencia de hemoparásitos en burros de la zona en estudio durante el 2017 y 2018.

Cuadro 1. Resultados del estudio de presencia de hemoparásitos en burros de los departamentos de Madriz y Nueva Segovia, 2016

\begin{tabular}{|c|c|c|c|c|c|c|c|c|c|c|c|c|c|}
\hline 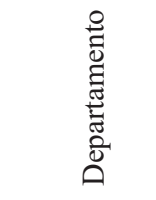 & 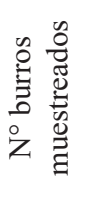 & 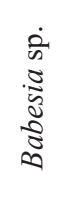 & 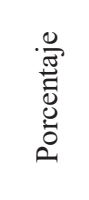 & 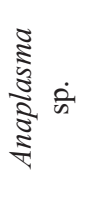 & 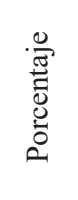 & 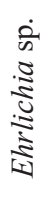 & 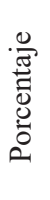 & 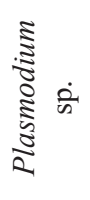 & 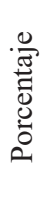 & 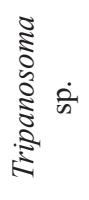 & 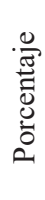 & 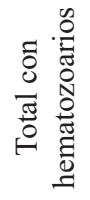 & 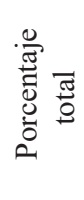 \\
\hline Madriz & 264 & 141 & 53.41 & 0 & 0.00 & 0 & 0 & 0 & 0 & 0 & 0 & 141 & 53.41 \\
\hline $\begin{array}{l}\text { Nueva Se- } \\
\text { govia }\end{array}$ & 415 & 181 & 43.61 & 24 & 5.78 & 0 & 0 & 0 & 0 & 0 & 0 & 205 & 49.40 \\
\hline Totales & 679 & 322 & 47.42 & 24 & 3.53 & 0 & 0 & 0 & 0 & 0 & 0 & 346 & 50.96 \\
\hline
\end{tabular}

Cuadro 2. Presencia de hemoparásitos en burros de los departamentos de Madriz y Nueva Segovia, 2017

\begin{tabular}{|c|c|c|c|c|c|c|c|c|c|c|c|c|c|}
\hline 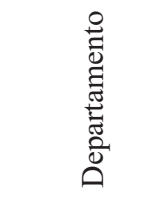 & 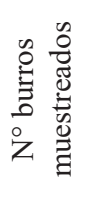 & 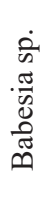 & 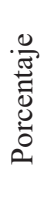 & 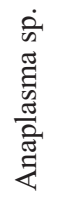 & 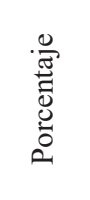 & 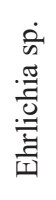 & 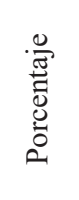 & 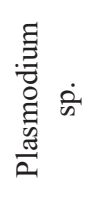 & 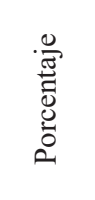 & 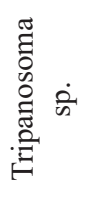 & 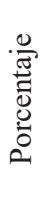 & 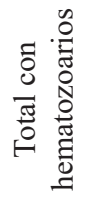 & 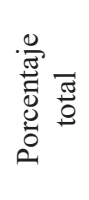 \\
\hline Madriz & 166 & 0 & 0 & 60 & 36.14 & 2 & 1.20 & 28 & 16.87 & 0 & 0 & 90 & 54.22 \\
\hline $\begin{array}{l}\text { Nueva Se- } \\
\text { govia }\end{array}$ & 83 & 0 & 0 & 13 & 15.66 & 0 & 0.00 & 0 & 0.00 & 0 & 0 & 13 & 15.66 \\
\hline Totales & 249 & 0 & 0 & 73 & 29.32 & 2 & 0.80 & 28 & 11.24 & 0 & 0 & 103 & 41.37 \\
\hline
\end{tabular}

Datos no publicados 
Al notar la diferencia en los resultados de ambos años sobre la prevalencia de Babesia sp., se realizó una entrevista al azar de cien tenedores de burros, preguntando los posibles motivos de no haberse presentado el segundo año al relevamiento, constatando que a un $75 \%$ de los propietarios, se les había muerto el burro, un $20 \%$ lo habían vendido, y un $5 \%$ explicaban otros motivos, como ausencia del lugar el día indicado, trabajo, otras tareas importantes.

Lo primero que llama la atención, es el abrupto descenso de los casos de Babesia $s p$ de un año a otro, que pasan de un $47.42 \%$ de burros afectados en el 2016 a $0 \%$ en el 2017. Lamentablemente, luego de las entrevistas telefónicas, constatamos que la prevalencia bajó lamentablemente por el fallecimiento o muerte de los burros afectados.

Si bien hay un descenso global de la presencia de hemoparásitos en burros de un año a otro de un 9.59\% (la prevalencia global baja de $50.96 \%$ a $41.37 \%$ ), se deben fundamentalmente al deceso de los portadores de Babesia $s p$, porque la presencia de Anaplasma $s p$ se incrementa de un año para otro en un $829 \%$, apareciendo además dos hemoparásitos que antes no se habían visualizado, Plasmodium $s p$ y Ehrlichia $s p$.

La presencia de Plasmodium sp es importante, no solamente porque es un elemento nuevo en el diagnóstico de hemoparásitos en burros (Anzuino, 2008; Quiroz, 2003), sino por el peligro inherente a la salud pública. Estudiando la geografía para ver porque se daba solo en algunos municipios de Somoto, Madriz y no se evidenciaba en Nueva Segovia, se descubrió que todos los casos se daban en la cuenca del Río Tapacale, afluente y principal raíz del Río Coco, y que en este se drenaban los efluentes del Hospital de Somoto.

\section{CONCLUSIONES}

Se registró la presencia de Babesia sp., Anaplasma sp., Ehrlichia sp. y Plasmodium sp., en los burros de la región.

Los hemoparásitos son el grupo de enfermedades más importante en los burros de Madriz y Nueva Segovia, con una prevalencia cercana al $50 \%$.

Las causas de muerte se deben mayormente a la falta de control de garrapatas en burros por parte de sus dueños, sumado a la falta de control y tratamiento de la hemoparasitosis.

Con el fallecimiento de los burros afectados por Babesia sp la Anaplasmosis pasa a ser la enfermedad mas relevante dentro de los hematozoarios, con más de $30 \%$ de prevalencia.

La presencia de Plasmodium sp no descrito hasta el momento en la literatura internacional es relevante, no solo porque afecta al $16.88 \%$ de los burros de las diferentes comarcas de la cuenca del Río Tapacale en Somoto, sino por los riesgos inherentes a la salud pública.

\section{RECOMENDACIÓN}

Se precisa capacitar a los dueños de los burros sobre manejo de garrapatas y hemoparásitos, así como en la gestión de recurso para su tratamiento.

\section{AGRADECIMIENTO}

Se agradece a Society for Protection Animal and Nature (SPANA) por el financiamiento otorgado para la realización del proyecto.

\section{REFERENCIAS BIBLIOGRÁFICAS}

Anzuino, J. 2008. Exotic infections. In Svendsen, ED; Duncan, J; Hadrill, D. 2008. The Professional Handbook of the Donkey, 4 ed. Whittet Books, Chapter 14.

Díaz M, SW; Talavera A, OG. 2017. Caracterización general de la población local de burros (Equus asinus) de las comunidades rurales de los departamentos del norte de Nicaragua, Madriz y Nueva Segovia, 2016. Tesis médico veterinario, Managua, NI. Universidad Nacional Agraria. 41 p.

Quiroz, H. 2003. Parasitología y enfermedades parasitarias de los animales domésticos, Editorial Limusa. 868 p.

Rimbaud, E; Sequeira, E; González, D; Mayorga, M. 2017. Prevalencia de hematozoarios y parásitos gastrointestinales en animales de trabajo en Nicaragua. Boletín de Parasitología 18(2):4. Disponible en http://www.hoy.com.ni/2016/10/30/\%ef\%bb\%bf\%ef\%bb\%bffuturo-dudoso-de-los-burros-en-nicaragua/

Rimbaud, E; Molina, C; Talavera, G; Sequeira, E. 2016. Caracterización de la población de burros en las comunidades de mosonte, Nueva Segovia, Nicaragua. Veterinaria Argentina XXXIII, 339.

Rimbaud, E. 2015. La problemática del burro en las Segovias: tradición, machete de trabajo o especie en extinción. Musunce. (90):31

Rodak, B. 2004. Hematología: fundamentos y aplicaciones clínicas. Médica Panamericana. Buenos Aires, AR. 